\title{
SUR LE MOMENT CYBERNÉTIQUE
}

\author{
Ronan Le RouX*
}

$\mathrm{L}^{\prime}$ ouvrage de Mathieu Triclot reprend quasiment au chapitre près sa thèse de doctorat soutenue fin 2006 à Lyon III et dirigée par Daniel Parrochia, habitué de la collection « Milieux ». Celle-ci s'enrichit ainsi d'un volume représentatif de l'une de ses thématiques usuelles, les rapports science-technique-société vus par une lorgnette philosophique. Le présent livre cherche principalement à articuler deux thèses: 1) il existe un « clivage essentiel » dans la représentation de l'information dès le stade d'élaboration de cette notion en contexte technologique; 2) ce clivage « constitue une des clefs d'intelligibilité majeure de l'histoire de la cybernétique» (p. 71). Une lecture semblable avait été proposée en 2005 par le physicien Freeman Dyson ${ }^{1}$.

La première des quatre parties du livre («L'idée de message ») présente l'émergence de la notion technologique d'information, dans le contexte de l'évolution des télécommunications aux États-Unis depuis la fin du XIX $x^{\mathrm{e}}$ siècle. L'approche peut ici être qualifiée d'externaliste, dans la mesure où elle introduit les élaborations théoriques comme des réponses à des besoins ou des solutions à des problèmes : le coût des télécommunications pour Shannon, la conduite de tir pour Wiener. L'auteur distingue deux conceptions de l'information derrière l'équivalence des formules mathématiques produites presque simultanément par ces deux grands noms: "L'information peut être représentée tantôt comme un code, une suite de symboles sans lien avec les choses, tantôt comme un signal, l'expression matérielle d'un agencement matériel » (p. 12). Ce clivage est présenté comme intrinsèque et irréductible.

La deuxième partie ( Ordinateur, esprit, cerveau ») explore des textes d'Alan Turing, John von Neumann et Warren McCulloch à la recherche des formes naissantes du traitement de l'information, et y retrouve la dichotomie code/signal. L'idée de machine logique, de software, se détache progressivement des considérations matérielles, mais la problématique de leur articulation demeure sous-jacente et travaille les textes fondateurs des représentants du traitement symbolique de l'information. L'auteur souligne que les interprétations courantes en histoire de l'informatique tendent à négliger ce point en formant l'illusion rétrospective que la séparation du logique et du matériel serait brusquement survenue comme un progrès évident et inéluctable. Cette partie est

* À propos de Mathieu Triclot, Le Moment cybernétique. La constitution de la notion d'information, Seyssel, Champ Vallon (Milieux), 2008, 425p. Ronan Le Roux est actuellement doctorant à l'École des hautes études en sciences sociales, au Centre Maurice Halbwachs (École normale supérieure, Centre Maurice-Halbwachs, 48, boulevard Jourdan, F-75014 Paris; ronan.le.roux@gmail.com).

1. Freeman J. Dyson, « The Tragic Tale of a Genius », The New York Review of Books, vol. 512, $\mathrm{n}^{\circ} 12-14,2005$. 
très marquée par des problématiques fondamentales de ce qui ne s'appelle alors pas encore les sciences cognitives.

La troisième partie («Cosmologie de l'information»), de façon symétrique, est consacrée à l'interprétation physicaliste de la notion d'information. Elle insiste particulièrement sur l'analogie entre information et entropie négative, et récapitule les étapes qui avaient vu les prémisses d'une élaboration de cette analogie dans le contexte de la physique, indépendamment des télécommunications ${ }^{2}$. Un chapitre présente les critiques épistémologiques adressées par Karl Popper et Charles Bennett à cette analogie. Cette troisième partie contient notamment une très intéressante analyse comparée des interprétations que Léon Brillouin et Norbert Wiener font du « démon de Maxwell ». Un chapitre final traite de la théorie des automates, que l'auteur inclut dans la cybernétique, donnant donc de celle-ci une définition large.

L'étude de Triclot n'apporte pas de matériau historique inédit, mais opère une synthèse d'éléments exploités dans les études existantes. Nous nous contenterons de formuler ici quelques perspectives critiques. Tout d'abord, l'interprétation générale défendue par l'auteur pose des problèmes. Concernant la première thèse (celle du « clivage »), un paradoxe subsiste sans être résolu (ni d'ailleurs à peine soulevé). Comment la cybernétique peut-elle être à la fois « clivage» (p. 11: « la notion d'information apparaît non pas une, mais clivée au sein du milieu technique. L'émergence du discours cybernétique de l'information peut être décrite comme une opération de clivage ») et " une extraordinaire opération d'unification du milieu technique sous les catégories informationnelles » (p. 72)? On ne comprend pas comment la notion d'information peut être, simultanément, unificatrice et intrinsèquement clivée. Qu'elle opère une synthèse des télécommunications, des asservissements et du calcul mécanique, en imposant à cette synthèse une partition entre deux représentations différentes, est un point de vue défendable, mais qui ne lève pas toutes les difficultés. S'il y a compatibilité technique par l'équivalence des formules de Shannon et de Wiener, comment pourrait-il y avoir incompatibilité ontologique ou simplement paradigmatique? Là où l'auteur semble voir une incommensurabilité entre le cas symbolique et le cas matériel («conflit irrémédiable », « point de blocage », « univers d'objets différents »), n'est-il pas tout simplement question d'une banale distinction entre deux cas, selon que l'on convienne ou non d'ajouter des paramètres pour tenir compte de la dimension physique du phénomène, du temps, des perturbations, etc.? S'il n'y a pas d'incommensurabilité, le " clivage » n'est donc pas intrinsèque à la notion d'information - par contre, il concerne sans doute les réflexions et interprétations divergentes de ceux qui y recourent -, ce que l'auteur, d'ailleurs, est bien amené à reconnaître à demi-mot (voir p. 26 et 213). S'il ne s'agit pas d'une confusion, nous ne trouvons pas que l'argumentation en dissipe l'impression de façon satisfaisante. Le commentaire de la théorie de Claude Shannon semble supposer que la compression d'un message implique que celui-ci soit composé de symboles (p. 26, 49), ou que la problématique du codage soit nécessairement « désincarnée ». Plus loin, la théorie des automates de John von Neumann est située du côté

2. On comparera avec la somme de Jérôme Segal, qui y ajoute le travail du statisticien Ronald Fischer (que Triclot ne mentionne pas, bien qu'il soit cité par Wiener): Jérôme SEGAL, Le Zéro et le Un. Une histoire de la notion scientifique d'information au XX siècle, Paris, Syllepse, 2003, chap. I. 
de la cybernétique, par conséquent du côté d'une représentation physicaliste de l'information soi-disant incompatible avec une représentation symbolique... La chose est peu compréhensible, étant donné le débouché que les langages formels représentent pour cette théorie; en fait, cela prouve de facto que la thèse d'une incompatibilité ou d'une incommensurabilité ne peut pas tenir en l'état.

Passons à ce que nous identifions comme la seconde thèse du livre: «le choix philosophique d'une représentation physicaliste [...] est au cœur du programme cybernétique [et] rend compte de l'extension de la discipline comme de son brusque déclin » (p. 12). Cette histoire paraît abordée du point de vue de l'histoire des sciences cognitives, avec un débat dont l'auteur, quoiqu'il en dise, ne semble pas avoir renouvelé les termes depuis la critique anti-connexionniste de Marvin Minsky en 1968 (voir p. 10): la cybernétique est considérée avant tout comme une science cognitive avortée. Cette perspective, pour légitime qu'elle soit, n'a pas lieu d'être exclusive, et si l'on veut la privilégier, il importe d'expliquer à quel titre et jusqu'à quel point. Car elle implique des jugements de valeur sur les autres manifestations historiques de la cybernétique. Et sur ce point, l'auteur risque un reproche voisin de celui qu'on pouvait adresser à Jean-Pierre Dupuy ${ }^{3}$ : construire son objet d'étude sur la base des seuls enjeux liés aux sciences cognitives, et négliger les rapports aux autres disciplines, considérés au mieux comme production de simples «métaphores » dont l'incidence épistémologique et les mécanismes de diffusion restent opaques. En admettant que la cybernétique soit une science ou un projet de science, elle n'est pas que cela, et n'a donc pas à être jugée uniquement en fonction des critères avec lesquels on juge habituellement des fortunes disciplinaires. Il nous paraît donc excessif d'affirmer que la cybernétique «a échoué de a à $\mathrm{z}$ » (p. 9). De même, fixer son « déclin » à la première moitié des années 1950 mériterait au minimum une confrontation à quelques données bibliométriques ${ }^{4}$. Enfin, en ce qui concerne « l'extension » de la cybernétique, l'auteur se contente de consacrer quelques pages à un "rendez-vous manqué » avec la biologie moléculaire; celle-ci se limite-t-elle donc à Max Delbrück? Qu'en est-il, par exemple, du modèle MWC (Jacques Monod - Jeffries Wyman - Jean-Pierre Changeux), auréolé d'un prix Nobel, qui inaugura une lignée de modèles de rétrocontrôles en génétique, en se réclamant explicitement de la cybernétique qui plus est? Et la physiologie? l'éthologie? l'économie? la psychologie? L'histoire de la cybernétique nécessite de nombreux autres chapitres, dans lesquels la question centrale ne sera pas toujours focalisée sur la notion d'information. Il n'y a pas que cette notion, matérialiste ou non, dans la cybernétique. Celle de feedback est rapidement escamotée dans la première partie du livre, réduite aux techniques électriques élaborées avant-guerre aux laboratoires Bell. Un historien des techniques y trouverait à redire. Ce n'est pas parce que les techniques de communication et de régulation partagent ou ont temporairement partagé un même formalisme

3. Jean-Pierre Dupuy, Aux origines des sciences cognitives, Paris, La Découverte, 1994.

4. À titre approximatif, sur les bases de référence Science Direct et Web of Science, le nombre total de publications scientifiques ou techniques comportant au moins une occurrence des termes «cybernetics », «cybernetic » ou «cybernétique » (sic) est en croissance nette et très régulière jusqu'aux années 1990. Le nombre de publications compris entre 1950 et 1970 représente environ $7 \%$ seulement du total jusqu'en 2007. 
(méthode fréquentielle) ou un même support physique qu'elles sont réductibles au même schématisme.

Finalement, c'est un préjugé conceptuel (la thèse du clivage) qui limite par avance une démarche qui se voulait une « histoire matérielle des sciences » (p. 12). Qu'il soit d'origine technologique (opposition software/hardware) ou philosophique (opposition idéalisme/matérialisme), on ne voit pas à quel titre cet a priori servirait d'étalon pour comprendre une histoire plus complexe et foisonnante que l'échantillon qu'en donne l'auteur. Dans le premier cas, ce serait une prédilection paradigmatique réductrice (en aucun cas l'histoire de l'informatique n'a lieu d'être privilégiée pour aborder l'histoire de la cybernétique); dans le deuxième cas, il est inutile d'insister sur les dégâts qu'une dichotomie scolastique peut infliger à l'écriture de l'histoire. S'il est légitime d'utiliser l'hypothèse d'un clivage (celui-là ou un autre) pour explorer l'histoire, il faut admettre qu'on ne peut jamais rendre compte de toute l'histoire sur cette seule base, et qu'en vertu même du caractère artificiel de toute grille de lecture, des faits peuvent échapper à l'hypothèse. Autrement dit, l'auteur présuppose que la seconde thèse s'appuie sur la première thèse, mais sans mettre son interprétation à l'épreuve d'un corpus plus étendu pour en préciser les limites. La plupart des critères de construction de la cybernétique en tant qu'objet historique et épistémologique restent en effet implicites ou non argumentés: l'auteur en dit peu sur les raisons pour lesquelles il opère certains choix et non d'autres. La confrontation aux essais de Katherine Hayles ou Céline Lafontaine est nécessaire mais non suffisante (Claus Pias, qui a défendu une thèse contraire à celle de l'auteur ${ }^{5}$, n'est pas cité). Pourquoi persister à miser sur la distinction code/signal (ou digital/analogique) pour essayer de définir de façon ultime et privilégiée la cybernétique, alors même que, de l'aveu de l'auteur, « il existe bien une hésitation structurelle au sein de la cybernétique entre [ces] deux représentations de l'information » (p. 215)? Triclot, en se référant à Ian Hacking, reconnaît le caractère « transversal » (p. 6) et « nomade » (p. 319) des concepts de la cybernétique; comment cette dernière peut-elle être, dans ce cas, associée exclusivement à une interprétation physicaliste? C'est, selon nous, un autre aspect du problème esquivé par l'auteur. Il est en effet courant, dans la modélisation biologique, que la schématisation fonctionnelle des rétrocontrôles (quoi d'autre, sinon cybernétique?) soit opposée aux approches physiciennes ${ }^{6}$.

La dernière partie (« Politique de l'information ») se focalise sur l'engagement pacifiste de Wiener dans le difficile contexte de la Guerre froide. L'auteur en distingue plusieurs formes, qui sont autant de tentatives pour lier une réflexion éthique à sa mise en œuvre effective: promouvoir un usage civil de la cybernétique et alerter l'opinion. On apprécie particulièrement la synthèse de travaux historiques récents apportant un éclairage contextuel conséquent - à commencer par la biographie de Wiener, dont

5. Claus PIAS, «Analog, digital, and the cybernetic illusion », Kybernetes, vol. 34, n 3-4, 2005, p. 543-549. Pias situe la cybernétique du côté de la représentation symbolique et dématérialisée de l'information.

6. Voir, par exemple, René Tном, Modèles mathématiques de la morphogenèse, Paris, Bourgois, 1980, p.145. 
on trouve le compte rendu dans ce numéro ${ }^{7}-$, doublée d'un soin mis à nuancer les positions (parfois ambiguës) de Wiener contre les interprétations caricaturales que certains essayistes semblent trouver vocation à produire ${ }^{8}$. Le chapitre commentant ses écrits « sociologiques » est excellent et justifie à lui seul l'acquisition du livre: l'analyse du dernier chapitre de Cybernetics, la comparaison des deux éditions de Cybernétique et société apportent le degré de finesse et de précision requis par ces textes si souvent mal lus. Le dernier chapitre, consacré à la "philosophie » wienérienne des techniques, est lui aussi très bienvenu. Ces thèmes mériteraient d'être développés dans des études spécifiques.

On regrette donc que l'auteur ait voulu donner à sa thèse du « clivage » une portée qui prétende couvrir l'ensemble des domaines concernés par la cybernétique, et non seulement les domaines technologiques et cognitifs pour lesquels elle peut conserver sa pertinence initiale?

7. Voir infra, p. 202-203.

8. Voir aussi notre compte rendu de Céline LAFOnTAINE, L'Empire cybernétique, Paris, Le Seuil, 2004, dans la Revue de synthèse, t. 128, n 3-4, 2007, p. 472-475.

9. Relativiser la portée de cette thèse au « clivage » nous semble ainsi un réquisit minimum légitime pour l'écriture des chapitres manquants de l'histoire de la cybernétique. Pour faire pleinement valoir ce point de vue, nous renvoyons à notre thèse en préparation: La Cybernétique en France, 1948-1970 (Paris, École des hautes études en sciences sociales). 